(O2014 IEEE. Personal use of this material is permitted. Permission from IEEE must be obtained for all other uses, in any current or future media, including reprinting/republishing this material for advertising or promotional purposes, creating new collective works, for resale or redistribution to servers or lists, or reuse of any copyrighted component of this work in other works. This is the author's version of an article that has been published in the conference proceedings. 


\title{
Simultaneous Localization of a Receiver and Mapping of Multipath Generating Geometry in Indoor Environments
}

\author{
Christian Gentner, Markus Ulmschneider, Rostislav Karásek, Armin Dammann \\ German Aerospace Center (DLR) \\ Institute of Communications and Navigation \\ Oberpfaffenhofen, 82234 Wessling, Germany \\ Email: \{Christian.Gentner, Markus.Ulmschneider, Rostislav.Karasek, Armin.Dammann\}@dlr.de
}

\begin{abstract}
This paper presents an algorithm that aims at combining the pre-existing and dominating applications of radio signals, namely communication, navigation and sensing of an environment into an integrated approach. In wireless propagation the transmitted signal is reflected and scattered by objects. Especially in indoor or urban scenarios, the signal reaching the receive antenna consists of multiple paths, called multipath. Multipath reception degrades the accuracy of the positioning device as long as the receiver is based on standard methods. With Channel-SLAM we introduced an algorithm which uses multipath components (MPCs) for positioning instead of mitigating them. In this paper, we show that MPCs allow us in addition to estimating the position of a receiver, to estimate the locations of reflecting surfaces and scatterers. We show that these estimations relate to floor plans of indoor environments. To verify the proposed algorithm, we evaluate the algorithm based on measurements using an ultra-wideband (UWB) system, the Decawaves DW1000 UWB transceiver.
\end{abstract}

\section{INTRODUCTION}

In wireless propagation the transmitted signal is affected by multipath propagation. Hence, the transmitted signal is reflected and scattered by objects and the signal reaching the receive antenna consists of multiple paths, called multipath. Multipath reception degrades the accuracy of the positioning device as long as the receiver is based on standard methods [1]. Strategies to mitigate multipath effects on the ranging estimate are in general based on the estimation of the channel impulse response (CIR) [2], [3].

In this paper, we consider a multipath assisted positioning approach [4]-[7]. Multipath assisted positioning approaches use multipath components (MPCs) for positioning instead of mitigating them. E.g. [4], [5], [7] treat MPCs as line-ofsight (LoS) signals from virtual transmitters (VTs) which are static during the movement of the receiver. Hence, positioning with only one physical transmitter is possible by using the MPCs for positioning. With Channel-SLAM we introduced a multipath assisted positioning algorithm which estimates simultaneously the positions of the receiver and the VTs based on the estimated parameters of the MPCs using a simultaneous localization and mapping (SLAM) approach. So the algorithm does not require any prior information such as room-layout or a database for fingerprinting. The only conditions to be fulfilled are the presence of a multipath environment, a moving receiver as well as prior knowledge of the initial receiver states, i.e. position and moving direction.

In this paper, we extend Channel-SLAM to exploit the geometry information of the surrounding area. In addition to estimating the position of a receiver, we infer valuable geometric information on the locations of reflectors and scatterers. Mapping the geometry layout may help to improve the position estimation or can be used for other positioning approaches like multipath-enhanced device-free localization algorithms [8]. We introduce an algorithm which allows to estimate the positions of scatterers and reflecting surfaces. However, the proposed algorithm is limited to scatterer locations and the location of reflecting surfaces which occurred from a single bounce reflection. Estimating surfaces which were interacting in a multiple wireless signal bounce scenario will be studied in a future research. To verify the proposed algorithm, we evaluate the algorithm based on measurements using an ultrawideband (UWB) system. We use the Decawaves DW1000 UWB transceiver which enables cost effective real-time positioning with high accuracy in the order of $10 \mathrm{~cm}$ in indoor and outdoor scenarios.

The paper is structured as follows. Section II introduces Channel-SLAM and the system model, thereafter Section III describes the estimation of the environment. Section IV evaluates the proposed algorithm by measurements using the UWB DW1000 module. Finally, Section V concludes the paper.

\section{CONCEPT OF ChAnNel-SLAM}

In this section, we introduce the concept of ChannelSLAM. First, we show in Section II-A how each MPC can be interpreted as a LoS signal from a VT to the receiver. Second, we summarize in Section II-B the derivation of the Channel-SLAM algorithm based on particle filtering.

\section{A. System model}

Fig. 1 shows a transmitted signal which is reflected on smooth surfaces and scattered at a lamp post. The transmitter has a fixed position and the receiver is moving on the indicated path. If we have a look on the reflected signal, indicated by the 


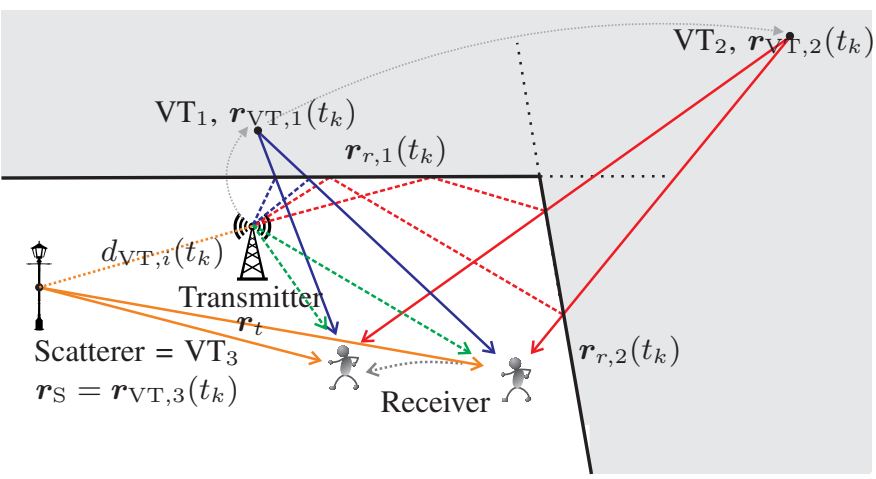

Fig. 1: The figure shows three multipath propagation scenarios: First scenario (blue): the transmitted signal is reflected on a reflecting surface. $\mathrm{VT}_{1}$ is defined by mirroring the physical transmitter position at the surface. Second scenario (red): the transmitted signal is reflected twice. $\mathrm{VT}_{2}$ is defined by mirroring the physical transmitter position at both surfaces. Third scenario (orange): the transmitted signal is scattered at a lamp post. $\mathrm{VT}_{3}$ is defined at the position of the lamp post.

blue lines, when the receiver is moving also the reflection point at the coordinates $\boldsymbol{r}_{r, 1}\left(t_{k}\right)$ is moving as well. If we mirror the physical transmitter position on the reflecting surface, we obtain the position $\boldsymbol{r}_{\mathrm{VT}, 1}$ of $\mathrm{VT}_{1}$ which is static during the receiver movement. The distance between $\mathrm{VT}_{1}$ and the receiver is equivalent to the propagation time of the reflected signal multiplied with the speed of light, hence,

$$
\begin{aligned}
d_{\mathrm{MPC}, 1} & =d_{\mathrm{TR}}\left(t_{k}\right)+d_{\mathrm{RU}}\left(t_{k}\right) \\
& =\left\|\boldsymbol{r}_{t}-\boldsymbol{r}_{r, 1}\left(t_{k}\right)\right\|+\left\|\boldsymbol{r}_{r, 1}\left(t_{k}\right)-\boldsymbol{r}_{\mathrm{u}}\left(t_{k}\right)\right\| \\
& =\left\|\boldsymbol{r}_{\mathrm{VT}, 1}-\boldsymbol{r}_{\mathrm{u}}\left(t_{k}\right)\right\| .
\end{aligned}
$$

This behavior can be extended to a multiple reflection scenario represented by the red lines. The transmitted signal is reflected two times. When the receiver is moving also the reflection point at the coordinates $\boldsymbol{r}_{r, 1}\left(t_{k}\right)$ and $\boldsymbol{r}_{r, 2}\left(t_{k}\right)$ is moving as well. Equivalently, the location of $\mathrm{VT}_{2}$ can be determined by mirroring the physical transmitter position at both reflecting surfaces, as indicated in Fig. 1. The distance between $\mathrm{VT}_{2}$ and the receiver is equivalent to the propagation time of the reflected signal multiplied with the speed of light. Thus, the signal reflected twice can also be interpreted as a direct signal from $\mathrm{VT}_{2}$ to the receiver.

Fig. 1 shows by the orange line a scenario where the transmitted signal is scattered at a lamp post. The propagation effect of scattering occurs if an electromagnetic wave impinges on an object and the energy is spread out in all directions [9]. Geometrically, the effect of scattering can be described as a fixed point at position $\boldsymbol{r}_{\mathrm{S}}$ in the pathway of the MPC. We define scatterer as $\mathrm{VT}_{3}$ at the position $\boldsymbol{r}_{\mathrm{S}}$ which is constant for all receiver positions for the MPC. Additionally, we treat $d_{\mathrm{VT}, 3}\left(t_{k}\right)>0$, the constant distance between physical transmitter and scatterer as an additional propagation distance associated to the MPC. Hence, the scattered signal can be in-

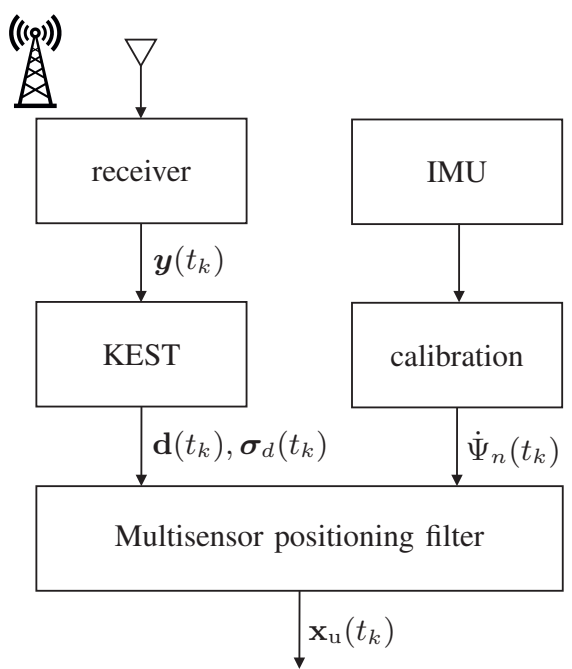

Fig. 2: System model consisting of a terrestrial receiver and an IMU.

terpreted as a direct signal from $\mathrm{VT}_{3}$ to the receiver, however, with a constant offset $d_{\mathrm{VT}, 3}\left(t_{k}\right)$. Scattering and diffraction can both be geometrically described as a fixed point at position $\boldsymbol{r}_{\mathrm{S}}$ in the pathway of the MPC and is considered as one model. Hence, unless otherwise stated, the description of scattering are equivalent for diffraction. This idea can be extended to all combinations of reflections, scattering and diffraction [7].

To summarize, the propagation path of the $i$-th MPC can be equivalently described as a direct path with propagation length $d_{\mathrm{MPC}, i}\left(t_{k}\right)$ between $\mathrm{VT}_{i}$ and the receiver plus an additional constant propagation length $d_{\mathrm{VT}, i}\left(t_{k}\right)$, hence,

$$
\begin{aligned}
d_{\mathrm{MPC}, \mathrm{i}} & =\tau_{i}\left(t_{k}\right) \cdot c \\
& =\left\|\boldsymbol{r}_{\mathrm{u}}\left(t_{k}\right)-\boldsymbol{r}_{\mathrm{VT}, i}\left(t_{k}\right)\right\|+d_{\mathrm{VT}, i}\left(t_{k}\right),
\end{aligned}
$$

where $c$ denotes the speed of light and $\boldsymbol{r}_{\mathrm{VT}, i}\left(t_{k}\right)$ the position of the $i$-th VT. Hence, the parameters representing the $i$-th VT can be described by

$$
\mathbf{x}_{\mathrm{VT}, i}\left(t_{k}\right)=\left[\boldsymbol{r}_{\mathrm{VT}, i}\left(t_{k}\right)^{T}, d_{\mathrm{VT}, i}\left(t_{k}\right)\right]^{T},
$$

where $\boldsymbol{r}_{\mathrm{VT}, i}\left(t_{k}\right)$ is the position of the $i$-th VT and $d_{\mathrm{VT}, i}\left(t_{k}\right)$ the additional propagation length. The additional propagation length is zero, i.e. $d_{\mathrm{VT}, i}\left(t_{k}\right)=0$, if only reflections occurred on the pathway between physical transmitter and receiver or greater than zero, i.e. $d_{\mathrm{VT}, i}\left(t_{k}\right)>0$, if the MPC is interacting with at least one scatterer. In general, $d_{\mathrm{VT}, i}\left(t_{k}\right) / c$ can be interpreted as a clock offset between the $i$-th VT and the physical transmitter. The position of the VTs and the additional propagation distances are constant over time. Nevertheless for notational convenience a time dependency on $t_{k}$ is introduced here. The model of (2) and (3) holds as well for the LoS path, hence, the physical transmitter position can also be interpreted and estimated as a VT. 


\section{B. Channel-SLAM derivation based on particle filtering}

Fig. 2 shows the available sensors together with the corresponding measurements. As shown on the left, we measure the sampled received signal from a local terrestrial transmitter. We use the dynamic multipath estimator named KEST [10], [11] for estimating and tracking multipath parameters. The estimated propagation path lengths $\hat{d}_{\mathrm{MPC}, i}\left(t_{k}\right)$ of all $N\left(t_{k}\right)$ MPCs, with $i=0, \ldots, N\left(t_{k}\right)-1$, of KEST are used as measurements

$$
\mathbf{d}\left(t_{k}\right)=\left[\hat{d}_{\mathrm{MPC}, 0}\left(t_{k}\right), \ldots, \hat{d}_{\mathrm{MPC}, N\left(t_{k}\right)-1}\left(t_{k}\right)\right]^{T},
$$

in the multisensor positioning filter. Additionally, an the heading change $\dot{\Psi}_{n}\left(t_{k}\right)$ and a movement indicator $m\left(t_{k}\right)$ of an IMU is used as input in the multisensor positioning filter.

Similarly to [7], the algorithm described in this paper follows a Bayesian approach which provides a methodology to optimally estimate parameters in non-stationary conditions. We assume a first order hidden Markov model and $\mathbf{x}\left(t_{k}\right)$ defines the state vector with

$$
\mathbf{x}\left(t_{k}\right)=\left[\mathbf{x}_{\mathrm{u}}\left(t_{k}\right)^{T}, \mathbf{x}_{\mathrm{VT}}\left(t_{k}\right)^{T}\right]^{T} .
$$

The state vector in (5) includes receiver states $\mathbf{x}_{\mathrm{u}}\left(t_{k}\right)$ and the VT states $\mathbf{x}_{\mathrm{VT}}\left(t_{k}\right)$, with

$$
\begin{aligned}
\mathbf{x}_{\mathrm{u}}\left(t_{k}\right) & =\left[\boldsymbol{r}_{\mathrm{u}}\left(t_{k}\right)^{T}, \boldsymbol{v}_{\mathrm{u}}\left(t_{k}\right)^{T}\right]^{T}, \\
\mathbf{x}_{\mathrm{VT}}\left(t_{k}\right) & =\left[\mathbf{x}_{\mathrm{VT}, 0}\left(t_{k}\right)^{T}, \ldots, \mathbf{x}_{\mathrm{VT}, N(-) 1}\left(t_{k}\right)^{T}\right]^{T}
\end{aligned}
$$

where $\boldsymbol{r}_{\mathrm{u}}\left(t_{k}\right)$ is the position of the receiver, the corresponding velocity $\boldsymbol{v}_{\mathrm{u}}\left(t_{k}\right)$ and the $i$-th VT state $\mathrm{x}_{\mathrm{VT}, i}\left(t_{k}\right)$ using (3).

We use a Rao-Blackwellized particle filter (RBPF) based on a sequential importance resampling (SIR) particle filter (PF) to estimate the marginalized posterior density $\mathrm{p}\left(\mathbf{x}_{\mathrm{u}}\left(t_{k}\right) \mid \mathbf{d}\left(t_{1: k}\right), \mathbf{u}\left(t_{1: k}\right)\right)$ with $N_{s}$ particles, with

$$
\begin{aligned}
& \mathrm{p}\left(\mathbf{x}_{\mathrm{u}}\left(t_{k}\right) \mid \mathbf{d}\left(t_{1: k}\right), \mathbf{u}\left(t_{1: k}\right)\right) \\
& \quad \approx \sum_{j=1}^{N_{s}} w^{(j)}\left(t_{k}\right) \delta\left(\mathbf{x}_{\mathrm{u}}\left(t_{k}\right)-\mathbf{x}_{\mathrm{u}}^{(j)}\left(t_{k}\right)\right),
\end{aligned}
$$

where the weights $w^{(j)}\left(t_{k}\right)$ are defined by

$$
w^{(j)}\left(t_{k}\right) \propto \prod_{i=0}^{N(t)-1} \sum_{a=1}^{N_{P, i, j}} w_{i}^{(j, a)}\left(t_{k}\right)
$$

with

$w_{i}^{(j, a)}\left(t_{k}\right) \propto w_{i}^{(j, a)}\left(t_{k-1}\right) \cdot \mathrm{p}\left(\hat{d}_{\mathrm{MPC}, i}\left(t_{k}\right) \mid \mathbf{x}_{\mathrm{u}}^{(j)}\left(t_{k}\right), \mathbf{x}_{\mathrm{VT}, i}^{(j, a)}\left(t_{k}\right)\right)$,

which is estimated according to [7] with PFs, where we use PFs to estimate the subspace representing the VTs inside a PF of (8). The likelihood $\mathrm{p}\left(\hat{d}_{\mathrm{MPC}, i}\left(t_{k}\right) \mid \mathbf{x}_{\mathrm{u}}^{(j)}\left(t_{k}\right), \mathbf{x}_{\mathrm{VT}, i}^{(j, a)}\left(t_{k}\right)\right)$ can be expressed as

$$
\mathrm{p}\left(\hat{d}_{\mathrm{MPC}, i}\left(t_{k}\right) \mid \mathbf{x}_{\mathrm{u}}^{(j)}\left(t_{k}\right), \mathbf{x}_{\mathrm{VT}, i}^{(j, a)}\left(t_{k}\right)\right)
$$

$$
=\frac{1}{\sqrt{2 \pi} \sigma_{d, \mathrm{MPC}, i}\left(t_{k}\right)} e^{-\frac{\left(\hat{d}_{\mathrm{MPC}, i}\left(t_{k}\right)-d_{\mathrm{MPC}, i}^{(j)}\left(t_{k}\right)\right)^{2}}{2 \sigma_{d, \mathrm{MPC}, i}^{2}\left(t_{k}\right)}},
$$

assuming the delay $\hat{d}_{\mathrm{MPC}, i}\left(t_{k}\right)$ to be independent Gaussian distributed and using (2) for the MPC $i$ where $\sigma_{d, i}^{2}$ denotes the variance obtained from KEST. Each particle $j=1 \ldots N_{s}$ consists of $N\left(t_{k}\right)$ subordinate particle filters (subPFs). Each subPF is represented by the particles $\mathbf{x}_{\mathrm{VT}, i}^{(j, a)}\left(t_{k}\right)$ with $a=$ $1, \ldots, N_{P, j, i}\left(t_{k}\right)$ where $N_{P, j, i}\left(t_{k}\right)$ stands for the number of particles in the $i$-th subPF with $i=0, \ldots, N\left(t_{k}\right)-1$, estimating $\mathbf{x}_{\mathrm{VT}, i}^{(j)}\left(t_{k}\right)$. Using subPFs for each VT allows to use different numbers of particles in each subPF.

For the transition prior $\mathrm{p}\left(\mathrm{x}_{\mathrm{u}}\left(t_{k}\right) \mid \mathbf{x}_{\mathrm{u}}^{(j)}\left(t_{k-1}\right), \mathbf{u}\left(t_{k-1}\right)\right)$ of the receiver state vector we use the transition model presented in [12] which incorporates the heading changes. Especially, we use the heading change $\dot{\Psi}_{n}\left(t_{k}\right)$ and a movement indicator $m\left(t_{k}\right)$ of the IMU as a control input $\mathbf{u}\left(t_{k}\right)=\left[\dot{\Psi}_{n}\left(t_{k}\right), m\left(t_{k}\right)\right]^{T}$ and is therefore directly integrated into the transition model. As mentioned before, the VT positions are stationary, hence, the transition prior of the $i$-th MPC is defined as $\mathrm{p}\left(x_{V T, i}\left(t_{k}\right) \mid x_{V T, i}^{(j)}\left(t_{k-1}\right)\right)=$ $\delta\left(\mathbf{x}_{\mathrm{VT}, i}\left(t_{k}\right)-\boldsymbol{x}_{V T, i}^{(j)}\left(t_{k-1}\right)\right)$. Please note, we assume independence among MPCs, i.e. propagation paths interacting with distinct objects, is based on the well-known uncorrelated scattering assumption in wireless propagation channel modelling [9].

In order to obtain a point estimate of the state, $\hat{\mathbf{x}}\left(t_{k}\right)=$ $\left[\hat{\mathbf{x}}_{u}\left(t_{k}\right)^{T}, \hat{\mathbf{x}}_{\mathrm{VT}}\left(t_{k}\right)^{T}\right]^{T}$, we use the minimum mean square error (MMSE) criterion which leads to the approximation of the MMSE for the receiver state

$$
\hat{\mathbf{x}}_{u}\left(t_{k}\right) \approx \sum_{j=1}^{N_{s}} w^{(j)}\left(t_{k}\right) \mathbf{x}_{\mathrm{u}}^{(j)}\left(t_{k}\right)
$$

and the $i$ th VT

$$
\hat{\mathbf{x}}_{\mathrm{VT}, \mathrm{i}}\left(t_{k}\right) \approx \sum_{j=1}^{N_{s}} w^{(j)}\left(t_{k}\right) \sum_{a=1}^{N_{P, j, i} t_{k}} w_{i}^{(j, a)}\left(t_{k}\right) \mathbf{x}_{\mathrm{VT}, i}^{(j, a)}\left(t_{k}\right) .
$$

Fig. 3 depicts the estimation procedure of the VTs. Compared to Fig. 1 we only show the VTs for the case of reflections and the physical transmitter. As mentioned before, the physical transmitter is also interpreted as a VT and estimated simultaneously during the receiver movement. At the first position, or whenever a new MPC is tracked, the VT states are initialized according to the estimated distance $\hat{d}_{\mathrm{MPC}, i}\left(t_{k}\right)$ for the $i$-th MPC, shown in Fig. 3a. The particles of the subPFs for the $i$-th MPC are initialized to hold (2), hence in a circle around the initial position $\boldsymbol{r}_{\mathrm{u}}\left(t_{k}\right)$. When the receiver is moving the uncertainty on the states of the VT is decreasing, see Fig. 3 b.

\section{ESTIMATION OF THE ROOM GEOMETRY}

In this section we introduce an algorithm to estimate the position of a reflecting surface for single bounce reflections. As introduced in Section II-A, the propagation path was 


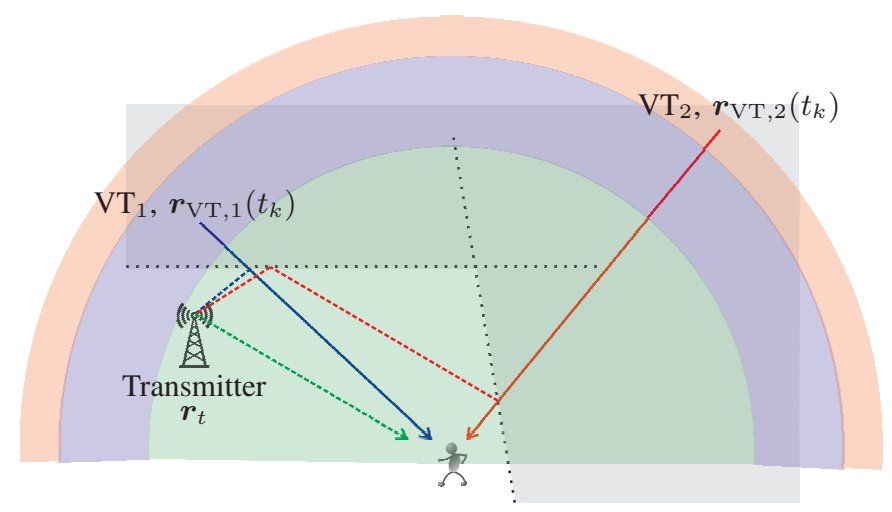

(a) First time step

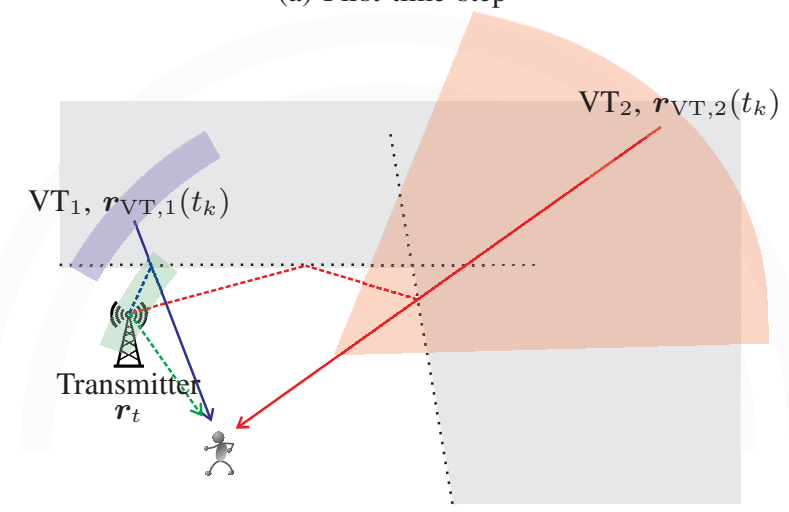

(b) Second time step

Fig. 3: Estimation process of the VT positions for two time steps. At the first time step the VT positions are initialized according to the estimated distance $\hat{d}_{\mathrm{MPC}, i}\left(t_{k}\right)$ of the $i$-th MPC in a circle around the estimated user position $\mathrm{x}_{\mathrm{u}}\left(t_{k}\right)$ using particles. During the receiver movement, the uncertainty on the VT positions decreases.

interacting on the pathway with a scatterer or was diffracted if the additional propagation distance of the $i$-MPC is greater than zero, hence, $d_{\mathrm{VT}, i}\left(t_{k}\right)>0$. Thus, the estimated VT position is either located on the scatter position itself or is located on a mirrored version of the scatterer, see [7]. If the additional propagation distance of the $i$-MPC is zero, hence, $d_{\mathrm{VT}, i}\left(t_{k}\right)=0$, only reflections occurred on the pathway between the transmitter and the receiver. In order to obtain the locations of the reflecting surfaces, the reflection points $\boldsymbol{r}_{r, 1}\left(t_{k}\right)$ and $\boldsymbol{r}_{r, 2}\left(t_{k}\right)$ have to be estimated, see Fig. 1. To obtain the position of the reflection points $\boldsymbol{r}_{r, 2}\left(t_{k}\right)$, the reflection points $\boldsymbol{r}_{r, 1}\left(t_{k}\right)$ have to be known. This paper concentrates on estimating the reflection points $\boldsymbol{r}_{r, 1}\left(t_{k}\right)$, estimating reflection points $\boldsymbol{r}_{r, 2}\left(t_{k}\right)$ will be studied in future research.

The calculation of the reflection points $\boldsymbol{r}_{r, 1}\left(t_{k}\right)$ is only possible if the position of the physical transmitter is known, or the estimation of the VT corresponding to the physical transmitter has converged. Additionally, the VT corresponding to the reflected path has to be converged. If the VTs are not converged, the uncertainty on the position of the reflection points is as high as the uncertainty on the positions of the

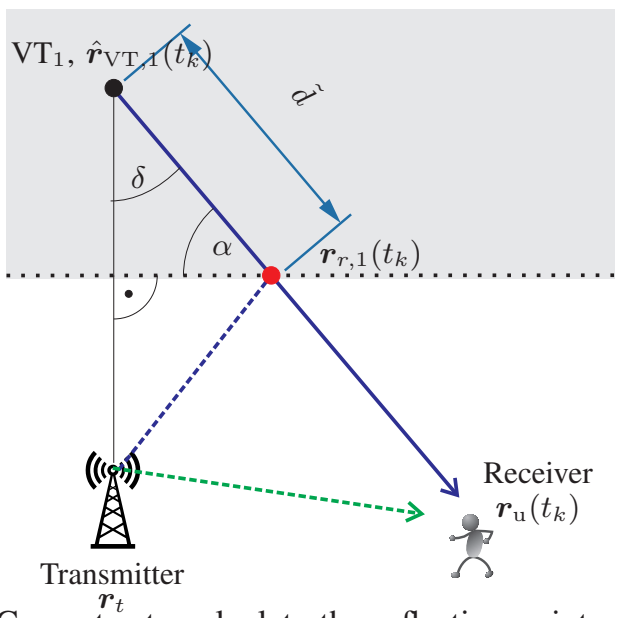

Fig. 4: Geometry to calculate the reflection points $\boldsymbol{r}_{r, 1}\left(t_{k}\right)$.

VTs, see Fig. 3b. So, the estimation of the reflection points $\hat{\boldsymbol{r}}_{r, 1}\left(t_{k}\right)$ is done as soon as the variance of the particle states is below the threshold $\sigma_{\mathrm{Thr}}^{2}$, with,

$$
\begin{aligned}
& \sigma_{\mathrm{MMSE}, i}^{2}= \\
& \quad \sum_{j=1}^{N_{s}} w^{(j)}\left(t_{k}\right) \sum_{a=1}^{N_{P, j, i} t_{k}} w_{i}^{(j, a)}\left(t_{k}\right)\left(\hat{\mathbf{x}}_{\mathrm{VT}, \mathrm{i}}\left(t_{k}\right)-\mathbf{x}_{\mathrm{VT}, i}^{(j, a)}\left(t_{k}\right)\right)^{2} \\
& \quad<\sigma_{\mathrm{Thr}}^{2},
\end{aligned}
$$

using (13).

If both VTs are converged, the estimated reflection points $\hat{\boldsymbol{r}}_{r, 1}\left(t_{k}\right)$ can be obtained, see also Fig. 4 , as

$$
\hat{\boldsymbol{r}}_{r, 1}\left(t_{k}\right)=\hat{\boldsymbol{r}}_{\mathrm{VT}, i}\left(t_{k}\right)+\tilde{d}[\cos (\delta), \sin (\delta)]^{T},
$$

using the vector between the transmitter position and $\mathrm{VT}_{i}$

$$
\boldsymbol{d}_{\mathrm{Tx}, \mathrm{VT}, \mathrm{i}}=\boldsymbol{r}_{t}-\hat{\boldsymbol{r}}_{\mathrm{VT}, i}\left(t_{k}\right),
$$

and the vector between the receiver position and $\mathrm{VT}_{i}$

$$
\boldsymbol{d}_{\mathrm{u}, \mathrm{VT}, \mathrm{i}}=\hat{\boldsymbol{r}}_{\mathrm{u}}\left(t_{k}\right)-\hat{\boldsymbol{r}}_{\mathrm{VT}, i}\left(t_{k}\right)=\left[\boldsymbol{d}_{\mathrm{x}, \mathrm{u}, \mathrm{VT}, \mathrm{i}}, \boldsymbol{d}_{\mathrm{y}, \mathrm{u}, \mathrm{VT}, \mathrm{i}}\right],
$$

with the angles

$$
\begin{aligned}
& \alpha=\arccos \left(\frac{\boldsymbol{d}_{\mathrm{u}, \mathrm{VT}, \mathrm{i}}^{T} \cdot \boldsymbol{d}_{\mathrm{Tx}, \mathrm{VT}, \mathrm{i}}}{\left\|\boldsymbol{d}_{\mathrm{u}, \mathrm{VT}, \mathrm{i}}\right\| \cdot\left\|\boldsymbol{d}_{\mathrm{Tx}, \mathrm{VT}, \mathrm{i}}\right\|}\right), \\
& \delta=\arctan \left(\boldsymbol{d}_{\mathrm{x}, \mathrm{u}, \mathrm{VT}, \mathrm{i}} / \boldsymbol{d}_{\mathrm{y}, \mathrm{u}, \mathrm{VT}, \mathrm{i}}\right) \text {, }
\end{aligned}
$$

and the distance between $\mathrm{VT}_{i}$ and the reflection point $\hat{\boldsymbol{r}}_{r, 1}\left(t_{k}\right)$

$$
\tilde{d}=\sqrt{f^{2}+(f \cdot \tan (\alpha))^{2}},
$$

with

$$
f=1 / 2\left\|\boldsymbol{d}_{\mathrm{Tx}, \mathrm{VT}, \mathrm{i}}\right\| .
$$

\section{EVALUATION BASED ON MEASUREMENT}

This section shows the performance of the described algorithm based an indoor measurement campaign. The measurements were conducted using an UWB system based on the Decawave DW1000 chip using a two-way-ranging method, see 


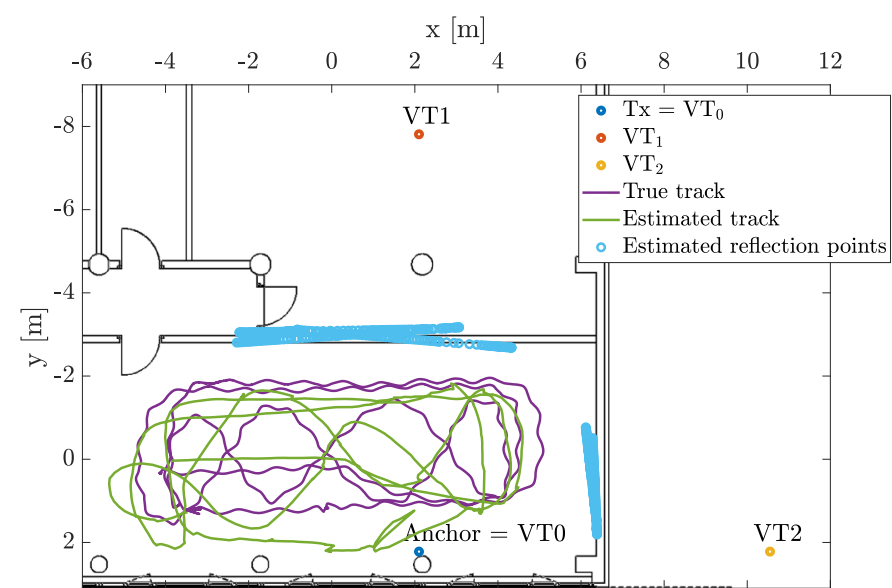

Fig. 5: Testing scenario with ground truth of the pedestrian movement and one realization of a proposed positioning algorithm including the estimation of the walls. Additionally, the three VTs are marked, $\mathrm{VT}_{0}-\mathrm{VT}_{2} . \mathrm{VT}_{0}$ is the position of the UWB anchor. Other VTs are caused by reflections from the room walls.

e.g., [13], [14]. The Decawave DW1000 chip can provide in addition to the ranging information the measured CIR. The measurement setup consists of one static UWB anchor and a mobile pedestrian carrying a hand-held device. The hand-held device includes an UWB tag, a Xsense IMU (MTI-G-700) and a laptop which stores the IMU and UWB measurement data. For the measurements, the UWB system is configured to a bandwidth of $500 \mathrm{MHz}$ and a carrier frequency of $3.5 \mathrm{GHz}$.

Fig. 5 shows the measurement scenario with the UWB anchor position indicated by $\mathrm{VT}_{0}$. The pedestrian was walking for $220 \mathrm{~s}$ on a track indicated by the purple line, with the starting-position in $\boldsymbol{r}_{\mathrm{u}}\left(t_{0}\right)=[0.3,-4.1]^{\mathrm{T}} \mathrm{m}$ and the endposition in $\boldsymbol{r}_{\mathrm{u}}\left(t_{\text {end }}\right)=[1.1,-3.7]^{\mathrm{T}} \mathrm{m}$. We used a Vicon motion capturer to track the movement of the pedestrians, hence, to obtain the ground truth. A reflector which was tracked by the Vicon motion capturer was attached to the handheld device. The Vicon motion capture system is capable of tracking the motion of the hand-held device within an accuracy below $1 \mathrm{~cm}$.

In order to exploit the multipath propagation for positioning, we have to estimate and track the MPCs over time. Hence, the accuracy of Channel-SLAM relies directly on the accuracy of the CIR estimations of KEST. Fig. 6 shows the estimation results of KEST for the CIR versus the pedestrian walking time in seconds where only the long visible paths are shown. We can observe that we have LoS condition, additionally, we can observe that some MPCs can be tracked for a long period of time. The first black line in Fig. 6 indicates the geometrical line-of-sight (GLoS) path delay, hence of $\mathrm{VT}_{0}$, which matches perfectly to the KEST estimates for the first path. Additionally, other MPCs can be tracked by KEST for a long time. The walls of the room act as a reflecting surface for the transmitted wireless signal. Hence, we can obtain the positions of $\mathrm{VT}_{1}$ and $\mathrm{VT}_{2}$ by mirroring the UWB anchor position at the walls, see

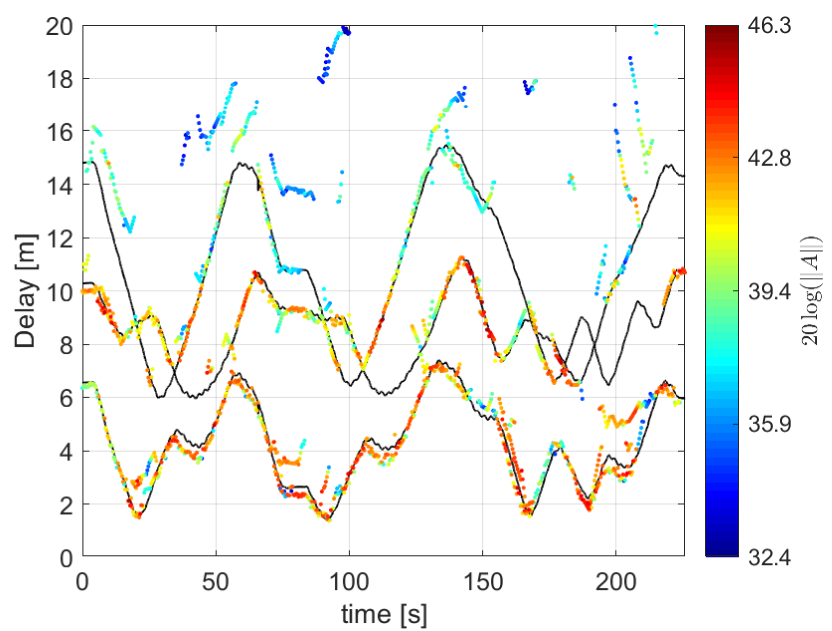

Fig. 6: Estimation results of KEST for the CIR versus the pedestrian walking times in seconds. Only long tracked paths are visualized. The black line indicates the theoretical propagation paths of the VTs, i.e. $\mathrm{VT}_{0}$ to $\mathrm{VT}_{2}$.

Fig. 5. The positions of the walls are measured using the Vicon motion tracker, thus, we are able to calculate the positions of $\mathrm{VT}_{1}$ and $\mathrm{VT}_{2}$. Based on the calculated VT positions, we are able to calculate the hypothetical propagation distances between these VTs and the moving pedestrian. We can see that they match the KEST estimates as indicated by the black lines in Fig. 6.

Channel-SLAM does not need any prior information except of the starting position. Hence, the UWB anchor position and the VT positions are unknown in Channel-SLAM. These positions are estimated simultaneously with the position of the moving pedestrian. Channel-SLAM has no information if the measured propagation paths correspond to the direct path, reflected or scattered paths. Hence, in order to calculate the reflection points as described in the last section, the algorithm assumes that the first path corresponds to the LoS path, however, with unknown UWB anchor position. Hence, after convergence of the different VT positions, Channel-SLAM calculates the reflection points.

Fig. 5 shows additionally in green the pedestrian position estimation result. We achieve a positioning accuracy of around $1 \mathrm{~m}$ in more than $95 \%$ of the cases. Additionally, the figure shows the estimation results of the reflecting points, hence the estimation of the walls, shown in blue. As we can see, the estimations fit to the floor-plan.

\section{CONCLUSIONS}

In this paper, we extend the multipath assisted positioning algorithm called Channel-SLAM. Channel-SLAM is an algorithm which uses multipath propagation for positioning instead of mitigating them. In this paper, we describe an algorithm that estimates the position of a receiver and the locations of reflecting surfaces and scatterers simultaneously. The proposed algorithm is evaluated based on measurements using an UWB 
system, the Decawaves DW1000 UWB transceiver. The evaluations of the measurements show that the estimated reflecting surfaces relate to the floor plan of the indoor measurement environment.

\section{REFERENCES}

[1] B. W. Parkinson and J. J. Spilker Jr., Global Positioning System: Theory and Applications, Vol. 1. American Institute of Aeronautics and Astronautics Inc., 1996.

[2] B. R. Townsend, P. C. Fenton, A. J. van Dierendonck, and D. J. R. V. Nee, "Performance Evaluation of the Multipath Estimating Delay Lock Loop," Journal of the Institute of Navigation, vol. 42, no. 3, pp. 503-514, Fall 1995.

[3] B. H. Fleury, M. Tschudin, R. Heddergott, D. Dahlhaus, and K. I. Pedersen, "Channel Parameter Estimation in Mobile Radio Environments using the SAGE Algorithm," IEEE J. Sel. Areas Commun., vol. 17, no. 3, pp. 434-450, Mar. 1999.

[4] P. Meissner, K. Witrisal, and K.Witrisal, "UWB for Robust Indoor Tracking: Weighting of Multipath Components for Efficient Estimation," IEEE Wireless Communications Letters, vol. 3, no. 5, pp. 501-504, Oct. 2014.

[5] E. Leitinger, F. Meyer, F. Hlawatsch, K. Witrisal, F. Tufvesson, and M. Z. Win, "A Belief Propagation Algorithm for Multipath-Based SLAM,' IEEE Trans. Wireless Commun., vol. 18, no. 12, pp. 56135629, 2019.

[6] T. Deissler and J. Thielecke, "Feature based Indoor Mapping using a Bat-Type UWB Radar," Sep. 2009, pp. 475-479.

[7] C. Gentner, T. Jost, W. Wang, S. Zhang, A. Dammann, and U.-C. Fiebig, "Multipath Assisted Positioning with Simultaneous Localization and Mapping," IEEE Trans. Wireless Commun., vol. 15, no. 9, pp. 61046117, Sep. 2016.

[8] M. Schmidhammer, C. Gentner, S. Sand, and U.-C. Fiebig, "MultipathEnhanced Device-Free Localization in Wideband Wireless Networks," arXiv:2010.04531, Oct. 2020.

[9] T. S. Rappaport, Wireless Communications - Principles and Practice. Prentice Hall, 1996.

[10] T. Jost, W. Wang, U.-C. Fiebig, and F. Pérez-Fontán, "Detection and Tracking of Mobile Propagation Channel Paths," IEEE Trans. Antennas Propag., vol. 60, no. 10, pp. 4875-4883, Oct. 2012.

[11] W. Wang, T. Jost, and A. Dammann, "Estimation and Modelling of NLoS Time-Variant Multipath for Localization Channel Model in Mobile Radios," Dec. 2010, pp. 1-6.

[12] C. Gentner, R. Pöhlmann, M. Ulmschneider, T. Jost, and S. Zhang, "Positioning using Terrestrial Multipath Signals and Inertial Sensors," Mobile Information Systems, Sep. 2017.

[13] C. Gentner and M. Ulmschneider, "Simultaneous Localization and Mapping for Pedestrians using Low-Cost Ultra-Wideband System and Gyroscope," Saporo, Japan, Sep. 2017.

[14] R. Karásek and C. Gentner, "Stochastic Data Association for Multipath Assisted Positioning Using a Single Transmitter," IEEE Access, vol. 8, 2020. 